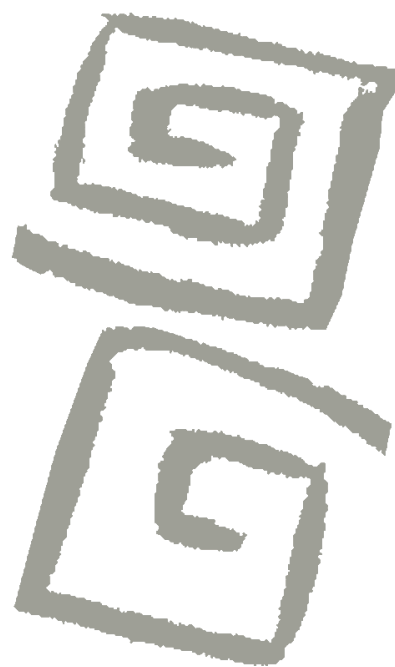

'Licenciada en Antropología. Magíster en Epidemiología, Gestión y Políticas de Salud. Becaria, CONICET, con sede en IDIP-CIC. Buenos Aires, Argentina. $\square$ (iD)

${ }^{2}$ Médica. Magíster en Epidemiología, Gestión y Políticas de Salud. Investigadora, IDIP-CIC Buenos Aires, Argentina. $\triangle$ iD

${ }^{3}$ Bioquímica. Investigadora, IDIP-CIC. Buenos Aires, Argentina. $\triangle$ (iD)

${ }^{4}$ Bioquímica. Jefa de Unidad de Docencia e Investigación, IDIP-CIC. Buenos Aires, Argentina. $\square$ (iD)

5Licenciada en Matemática. Investigadora, IDIP-CIC Buenos Aires, Argentina. $\triangle$ iD

${ }^{6}$ Licenciada en Nutrición. Personal de Apoyo, CIC, con sede en IDIP-CIC. Buenos Aires, Argentina. $\square$ (iD

${ }^{7}$ Médica. Personal de Apoyo, Comisión de Investigaciones Científicas, con sede en IDIPCIC. Buenos Aires, Argentina. $\triangle$ iD

${ }^{8}$ Médico. Doctor en Medicina. Responsable del Área de Planificación y Gestión, IDIP-CIC, Buenos Aires, Argentina. $\square$ iD

\section{Estudio cuali-cuantitativo del estado nutricional y la alimentación en niños de 1 a 3 años de familias de bajos recursos en dos grupos poblacionales con diferentes actividades productivas (Buenos Aires, Argentina), 2007-2008}

\author{
Quali-quantitative study of nutritional status and eating patterns \\ in children aged 1-3 years from low-income families in two \\ population groups with different productive activities (Buenos \\ Aires, Argentina), 2007-2008
}

Lorena Pasarin', Mariana A. Falivene'2, Liliana Disalvo ${ }^{3}$, Ana Varea ${ }^{4}$, María C. Apezteguía ${ }^{5}$, Agustina Malpeli ${ }^{6}$, Marisa Sala 7 , Horacio F. González ${ }^{8}$

RESUMEN El objetivo de este artículo es describir el estado nutricional y los patrones de alimentación de niños de 1 a 3 años de familias de bajos recursos, que residen en dos áreas con diferentes actividades productivas: producción primaria y de bienes y servicios. Se realizó un estudio descriptivo de corte transversal, con metodología cuali-cuantitativa; se evaluó el estado nutricional antropométrico y bioquímico, ingesta alimentaria, características económicas y sociodemográficas, prácticas alimentarias y representaciones. Los resultados muestran que los niños del área de producción primaria presentaron menor prevalencia de anemia y deficiencia de hierro. Asimismo tuvieron un consumo superior de energía, calcio, zinc, vitamina A y proteínas y una mayor diversidad y calidad en el consumo de alimentos. Podemos concluir que el contexto geográfico de las familias que residen cerca de fuentes de producción primaria favorecería la interacción con individuos ajenos a la familia, ampliando la red social informal y el acceso a alimentos de mejor calidad nutricional.

PALABRAS CLAVES Estado Nutricional; Preferencias Alimentarias; Niños; Anemia; Zinc; Argentina.

ABSTRACT The aim of this article is to describe the nutritional status and eating patterns of children aged 1-3 years from low-income families who reside in areas with different productive activities: primary production and production of goods and services. A descriptive cross-sectional study was performed with a qualitative and quantitative methodology, evaluating anthropometric and biochemical nutritional status, food intake, economic and demographic characteristics, dietary practices and representations. The results show that children from areas of primary production had a lower prevalence of anemia and iron deficiency. They also had a higher consumption of energy, calcium, zinc, vitamin A and protein and a greater diversity and quality in food consumption. We can conclude that the geographical context of families closer to sources of primary production favors interaction with individuals outside of the family, expanding both the informal social network and access to better quality nutritional food.

KEY WORDS Nutritional Status; Food Preferences; Child; Anemia; Zinc; Argentina. 


\section{INTRODUCCIÓN}

El estado nutricional de los niños menores de cinco años es un signo sensible del estado de salud de la población de un país, así como de la situación económica, y se encuentra vinculado a los contextos geográficos, la disponibilidad de recursos económicos, los tipos de actividades productivas realizadas por las poblaciones y, especialmente, al acceso y disponibilidad de los alimentos y a la calidad de lo que se consume $\mathrm{i}^{(1,2,3,4)}$. En este sentido, varios trabajos compararon las características poblacionales y, para ello, las diferenciaron en urbanas y rurales, haciendo referencia a la zona rural como la más desfavorable, en la que la producción de alimentos y la accesibilidad a estos es limitada ${ }^{(5,6)}$. Sin embargo, al estratificar las áreas rurales y urbanas según los recursos económicos disponibles, las diferencias en cuanto al estado nutricional de los niños ponen en evidencia que la malnutrición no está únicamente ligada a la población rural(5).

La disponibilidad de los alimentos y la diversidad de la dieta de los niños no solo es un problema económico, sino también un producto de las características del lugar donde habitan ${ }^{(7)}$. Las altas prevalencias de anemia y malnutrición en niños no disminuyen por vivir en un entorno urbano ${ }^{(8,9,10)}$. Aun así, en términos generales, se cree que los niños que habitan en las zonas urbanas tienen mejores condiciones de vida que sus contrapartes rurales; sin embargo, la proximidad física a un tipo de servicio en las grandes zonas urbanas no garantiza necesariamente el acceso a ese servicio $^{(11)}$. Varios trabajos han demostrado que, en muchos países, los niños que viven en la pobreza urbana tienen condiciones tan malas o peores que las de los niños que viven en la pobreza rural, en términos del peso para la talla o de la mortalidad en menores de 5 años ${ }^{(8,12)}$.

Dadas las crecientes desigualdades sociales, existe la necesidad de desarrollar abordajes de estudio que permitan evidenciar estas diferencias en los contextos de la salud, así como sus causas, en diferentes grupos poblacionales. Conocer las características de cada grupo poblacional permitirá comprender de qué manera las diferentes actividades productivas podrían influir en el acceso y la disponibilidad de los alimentos, así como en la calidad de lo consumido ${ }^{(2,13)}$. Todo ello plantea la necesidad de un abordaje interdisciplinario sobre el estado nutricional de los niños que viven en áreas donde las actividades productivas son diferentes.

El contexto del presente estudio, la provincia de Buenos Aires (Argentina), es el sector más poblado del país. Presenta una situación particular ya que su amplitud geográfica determina que la distribución, tanto de la densidad poblacional como de las actividades productivas, sea muy diversa. Existen zonas donde se desarrollan actividades de explotación primaria y secundaria que representan alrededor del $40 \%$ de la producción agrícola (trigo, maíz, girasol y cebada) y el $37 \%$ de las existencias bovinas del país, con baja densidad de población. En otras localidades se realizan actividades industriales y administrativas, con producción de servicios en los sectores de la construcción, electricidad, gas y agua, establecimientos financieros, seguros, o bienes inmuebles, y concentran el mayor porcentaje de población ${ }^{(14)}$.

El objetivo de este trabajo es describir el estado nutricional (antropométrico y bioquímico) y los patrones de alimentación de niños de 1 a 3 años de familias de bajos recursos, que residen en dos áreas con diferentes actividades productivas en la provincia de Buenos Aires (Argentina): un grupo que reside en áreas de actividades de producción primaria donde la actividad agrícolaganadera es la predominante, y un grupo que reside en áreas cuya actividad principal es la producción de bienes y servicios.

\section{METODOLOGÍA}

\section{Diseño}

Se realizó un estudio analítico de corte transversal, con abordaje cuantitativo y cualitativo, durante los años 2007 y 2008. 


\section{Revisión ética}

El protocolo de investigación y el consentimiento informado fueron aprobados por el Comité de Revisión de Protocolos de Investigación (CIRPI) del Instituto de Desarrollo e Investigaciones Pediátricas "Prof. Dr. Fernando E. Viteri" (IDIP), Hospital de Niños Sor María Ludovica (La Plata, Buenos Aires, Argentina) en el año 2007.

Se solicitó el consentimiento informado a todos los padres y/o tutores de los niños participantes, respetando la confidencialidad y el anonimato de la información brindada por ellos y/o obtenida en esta investigación.

\section{Población y Área}

El muestreo fue intencional. Se evaluaron niños sanos de 1 a 3 años de edad, asistidos en el sector público de salud en seis localidades de la provincia de Buenos Aires. Se excluyeron del estudio niños con enfermedades crónicas diagnosticadas, o que padecieran enfermedades agudas y/o infecciosas en el momento del estudio, y aquellos cuyos padres no aceptaron participar.

El grupo del área de producción de bienes y servicios se compuso por una muestra de niños residentes en Arturo Seguí, una localidad suburbana ubicada a $20 \mathrm{~km}$ de la ciudad de La Plata, capital de la provincia. El grupo del área de producción primaria comprendió una muestra de niños residentes en las localidades de Ayacucho, Balcarce, Dolores, Las Flores y Rauch, ubicadas en el interior de la provincia de Buenos Aires a más $150 \mathrm{~km}$ de la ciudad capital. La base económica dominante de estas localidades es la ganadería (cría) y agricultura ${ }^{(15)}$.

El trabajo en terreno fue realizado durante el período noviembre 2007 a mayo de 2008. La convocatoria fue organizada por el equipo de trabajo de cada municipio, decidiendo la/s opción/es más adecuada/s a fin de facilitar la participación voluntaria. La evaluación en terreno se concretó en los centros de salud y/o hospitales de las localidades incluidas en la investigación. Para las entrevistas etnográficas se incluyó la visita a los domicilios particulares.

\section{Cálculo del tamaño muestral}

Se determinó el tamaño muestral para poder detectar una diferencia del $8 \%$ de la prevalencia de anemia entre ambas regiones, con un nivel de significación $\alpha=0,05$ y una potencia $\beta=0,80$. Se calculó que era necesario encuestar, al menos, a 79 niños en cada región. Como información de base se tomó la Encuesta Nacional de Nutrición y Salud $(\text { ENNyS })^{(16)}$.

\section{Técnicas e instrumentos de recolección de datos}

A todos los niños se les tomó una muestra de sangre venosa en ayunas para determinar la hemoglobina, ferritina y zinc. La hemoglobina se determinó utilizando un contador hematológico automatizado (ABX Pentra 60), la ferritina se determinó por inmunoensayo utilizando un sistema automatizado de quimioluminiscencia Access Beckman Coulter y el zinc se determinó por espectrofotometría de absorción atómica por Ilama (AA-6200 Shimadzu). Se definieron las variables anemia $(\mathrm{Hb}<11 \mathrm{~g} / \mathrm{dl})^{(16)}$, deficiencia de hierro (ferritina sérica $<12 \mathrm{ng} / \mathrm{ml})^{(17)}$ y deficiencia de zinc (zinc sérico $<70 \mu \mathrm{g} / \mathrm{dl}$ ) ${ }^{(18)}$.

En terreno se obtuvieron las medidas de peso y talla con técnicas estándar ${ }^{(19)}$. Los participantes fueron pesados con balanza electrónica digital modelo Tanita UM-061 con divisiones de 100 g y capacidad máxima de $150 \mathrm{~kg}$. La estatura fue tomada con tallímetro portátil Seca con graduación de $1 \mathrm{~mm}$. Las mediciones fueron realizadas en cada localidad por nutricionistas previamente entrenados en una jornada de estandarización según la técnica de $\mathrm{Habitch}^{(20)}$. El error de medición de talla fue de $0,41 \mathrm{~cm}$.

Se analizaron los indicadores: peso/edad, talla/edad e índice de masa corporal (IMC) y se evaluaron con las tablas propuestas por la Organización Mundial de la Salud $\left(\mathrm{OMS}^{(19)}\right.$. 
Para la clasificación nutricional se usó: baja talla para la edad $<-2$ score $Z$ de talla/edad, bajo peso para la edad $<-2$ score $Z$ peso/ edad y obesidad $>+2,0$ score $Z$ según IMC.

Para evaluar la ingesta alimentaria se realizó un recordatorio de 24 horas al adulto responsable del cuidado de cada niño, según la técnica de múltiples pasos ${ }^{(21)}$. El recordatorio se realizó de modo de que estuvieran representados de forma equivalente todos los días de la semana, excepto cuando la alimentación del día anterior de estos niños se viera afectada por enfermedad. Para estandarizar cantidades y medidas se utilizaron kits de réplicas y modelos visuales de alimentos más consumidos (Fornax SRL-Food Replicas, Rosario, Argentina). Las cantidades de alimentos, bebidas y suplementos reportados fueron traducidos a nutrientes utilizando las tablas de composición química del United States Department of Agriculture (USDA) ${ }^{(22)}$ vinculadas a planillas especialmente diseñadas para tal fin en Access. Los nutrientes evaluados fueron: energía (kcal), proteínas (g), vitamina $A$ ( $\mu$ gr RE), folatos $(\mathrm{mg})$, calcio $(\mathrm{mg})$, hierro (mg) y zinc (mg).

Se realizó un cuestionario sobre características sociodemográficas y económicas del hogar para determinar porcentaje de hogares con necesidades básicas insatisfechas según los indicadores del Instituto Nacional de Estadística y Censos (INDEC) (23) de los años de estudio, edad del niño y de la madre, y trabajo y escolaridad materna (menos de primario completo corresponde a menos de 7 años de escolaridad; primario completo, a 7 años de escolaridad; y más de primario completo, a más de 7 años de escolaridad).

Asimismo, en todas las localidades se realizaron entrevistas semiestructuradas con metodología cualitativa buscando obtener información sobre las prácticas alimentarias y sus representaciones. Las entrevistas fueron realizadas a las madres de los niños participantes, tuvieron una duración promedio de una hora cada una y fueron registradas mediante grabador digital.

\section{Análisis}

Para el procesamiento de los datos cuantitativos ser utilizó el SPSS 18 para Windows. Las concentraciones de ferritina se expresaron como medias geométricas con intervalos de confianza del $95 \%$, ya que la distribución poblacional de esta variable no es normal. Se utilizaron los test de Mann-Whitney y chi-cuadrado para comparar medias y prevalencias respectivamente.

Se ajustaron modelos de regresión logística para prevalencia de anemia y de deficiencia de hierro que incluían región como variable dependiente y edad, sexo, educación materna y necesidades básicas insatisfechas como variables independientes, para evaluar la confusión. Como resultado, se calcularon los odds ratio (OR) corregidos por el modelo.

La información obtenida en las entrevistas cualitativas fue transcripta textualmente y procesada en Word. El material resultante fue incorporado al software NVivo, que permite organizar la información contenida en el corpus discursivo seleccionando unidades y vinculándolas a "nodos", a los que se asociaron categorías significativas de la alimentación en los contextos seleccionados para el estudio. Se realizó un análisis de las referencias y su frecuencia y se estableció un conjunto de nodos y sub-nodos que hicieron posible la organización del material a los fines de la descripción propuesta. Posteriormente, se procedió a registrar las recurrencias y diferencias en las apreciaciones y las opiniones de los entrevistados.

\section{RESULTADOS}

Se evaluaron 88 niños del área de producción de bienes y servicios y 114 del área de producción primaria. Ambas poblaciones fueron comparables según las variables consideradas, excepto en el nivel de educación materno que fue significativamente mayor en el área de producción primaria (Cuadro 1). Si bien el porcentaje de familias con necesidades básicas insatisfechas fue comparable en ambas 
Cuadro 1. Características socioeconómicas del área de producción de bienes y servicios y del área de producción primaria $(n=202)$. Provincia de Buenos Aires, Argentina, 2007-2008.

$\begin{array}{lcc}\text { Características socioeconómicas } & \begin{array}{c}\text { Área de producción de } \\ \text { bienes y servicios } \\ (\mathbf{n}=88)\end{array} & \begin{array}{c}\text { Área de producción } \\ \text { primaria } \\ (\mathbf{n}=\mathbf{1 1 4})\end{array} \\ \text { Edad de los niños (años) (media } \pm \text { DS) } & 1,6 \pm 0,4 & 1,7 \pm 0,5 \\ \text { Edad materna (años) (media } \pm \text { DS) } & 26,2 \pm 6,4 & 26,3 \pm 6,6 \\ \text { Necesidades básicas insatisfechas (\%) } & 40,9 & 38,9 \\ \text { Nivel educativo materno (\%) } & & \\ \quad \text { Menos de primario completo } & 44,2 & 17,3 \\ \quad \text { Primario completo } & 27,9 & 48,2 \\ \quad \text { Más de primario completo } & 27,9 & 27,5 \\ \text { Trabajo materno (\%) } & 31,8 & \\ & & \\ \text { Fuente: Elaboración propia. } & & \\ { }^{1} p=0,000 & & \end{array}$

regiones, las viviendas del área de producción de bienes y servicios no contaban con agua corriente ni con sistema de desagüe cloacal.

En cuanto a la actividad del jefe del hogar, en el área de producción de bienes y servicios predominó el trabajo informal o "changas" (albañiles, corte de pasto, trabajos de floricultura). Las mujeres eran amas de casa o trabajaban ocasionalmente como empleadas domésticas. En el área de producción primaria los varones jefes de hogar tenían trabajo estable como peones de campo (apicultores, leñadores, alambradores, etc.), empleados de fábricas o empleados municipales. Las madres no trabajaban fuera de su hogar o se desempeñaban como empleadas domésticas o cuidaban personas en casas de familia.

Cuadro 2. Prevalencia de anemia y deficiencia de hierro y zinc en el área de bienes y servicios y en el áreas de producción primaria. Provincia de Buenos Aires, Argentina, 2007-2008.

\begin{tabular}{|c|c|c|c|c|c|}
\hline \multirow[t]{2}{*}{ Prevalencia } & \multicolumn{2}{|c|}{$\begin{array}{l}\text { Área de producción de } \\
\text { bienes y servicios } \\
(n=88)\end{array}$} & \multicolumn{2}{|c|}{$\begin{array}{l}\text { Área de producción } \\
\text { primaria } \\
(\mathrm{n}=114)\end{array}$} & \multirow[t]{2}{*}{ Valor de $p$} \\
\hline & (\%) & IC95\% & $(\%)$ & IC95\% & \\
\hline Anemia & 42,0 & 31,$7 ; 52,3$ & 25,9 & 17,$7 ; 34,2$ & 0,017 \\
\hline Deficiencia de hierro & 48,3 & 37,$8 ; 58,8$ & 32,3 & $22.9 ; 41,6$ & 0,027 \\
\hline Deficiencia de zinc & 10,3 & 3,$9 ; 16,7$ & 6,5 & 2,$6 ; 10,2$ & 0,338 \\
\hline
\end{tabular}


Cuadro 3. Modelo de regresión logística para la prevalencia de anemia y deficiencia de hierro (n=202). Provincia de Buenos Aires, Argentina, 2007-2008.

\begin{tabular}{|c|c|c|c|c|c|c|}
\hline \multirow[t]{2}{*}{ Factor de riesgo } & \multicolumn{2}{|c|}{ Anemia $^{1}$} & \multirow[t]{2}{*}{ Valor de $p$} & \multicolumn{2}{|c|}{ Deficiencia de hierro $^{2}$} & \multirow[t]{2}{*}{ Valor de $p$} \\
\hline & OR & IC95\% & & OR & IC95\% & \\
\hline $\begin{array}{l}\text { Área de producción de } \\
\text { bienes y servicios }\end{array}$ & 2,035 & 1,$094 ; 3,784$ & 0,025 & 2,427 & 1,$284 ; 4,586$ & 0,006 \\
\hline Edad & 0,460 & 0,$224 ; 0,944$ & 0,034 & 0,561 & 0,$276 ; 1,141$ & 0,111 \\
\hline Sexo & 0,806 & 0,$428 ; 1,519$ & 0,505 & 2,009 & 1,$057 ; 3,819$ & 0,033 \\
\hline
\end{tabular}

Con relación a los parámetros bioquímicos, los valores medios de hemoglobina y ferritina fueron inferiores en el área de producción de bienes y servicios. Este grupo presentaba mayores prevalencias de anemia y de deficiencia de hierro. No se observaron diferencias con respecto al zinc (Cuadro 2). La aplicación del modelo de regresión logística mostró que, aun ajustando por las variables de confusión, la prevalencia de anemia estuvo asociada al área de producción de bienes y servicios y a la edad de los niños. Para la deficiencia de hierro se observó una asociación entre esta misma área y el sexo masculino (Cuadro 3).

La evaluación antropométrica mostró que los problemas prevalentes fueron baja talla $(13,6 \%$ vs. $15,9 \%)$ y obesidad $(15,9 \%$ vs. $16,8 \%$ ), en el área de producción de bienes y servicios y el área de producción primaria respectivamente, sin diferencias significativas entre ambos grupos.

Por otro lado, los resultados de la encuesta alimentaria mostraron que los valores de consumo diario de energía, calcio, zinc, vitamina A y proteínas fueron significativamente más bajos en el área de producción de bienes y servicios (Cuadro 4).

A partir de las entrevistas semiestructuradas se pudo observar una gran similitud entre las familias de los dos grupos. Los grupos domésticos estuvieron conformados por familias nucleares y monoparentales. Sus integrantes eran de procedencia local o de pueblos cercanos. Algunos grupos domésticos estaban integrados por individuos provenientes de provincias del interior del país o de países limítrofes, como Paraguay o Bolivia. Las compras para abastecimiento de alimentos, en el área de producción primaria, se hacían en comercios cercanos a los hogares (mercados, almacenes y carnicerías) mientras que, en el área de producción de bienes y servicios, las familias realizaban compras generales en grandes supermercados debido a la diferencia de precios con los comercios cercanos al hogar, práctica que no fue mencionada en el otro grupo.

En relación con la producción propia de alimentos, en el área de producción primaria algunas de las unidades domésticas refirieron contar con la producción de alimentos de huerta y plantas frutales, recurso no referido en el área de producción de bienes y servicios. En ambos grupos se señaló la cría de gallinas y pollos para el consumo de carne y producción de huevos. En el área de producción primaria algunos alimentos se obtenían por medio de trueque o canje (carnes, en general) y regalos de familiares y vecinos (frutas, verduras, carnes y/o huevos).

Al considerar el tipo de comidas ambos grupos mencionaron el consumo de ciertos platos "de olla" (puchero, guiso de arroz, 
Cuadro 4. Datos de encuesta alimentaria del área de producción de bienes y servicios y del área de producción primaria $(n=202)$. Provincia de Buenos Aires, Argentina, 2007-2008.

\begin{tabular}{|c|c|c|c|c|c|}
\hline \multirow[t]{2}{*}{ Nutrientes } & \multicolumn{2}{|c|}{$\begin{array}{l}\text { Área de producción de } \\
\text { bienes y servicios } \\
(\mathbf{n}=88)\end{array}$} & \multicolumn{2}{|c|}{$\begin{array}{l}\text { Área de producción } \\
\text { primaria } \\
(n=114)\end{array}$} & \multirow[t]{2}{*}{ Valor de $p$} \\
\hline & Mediana & $\begin{array}{l}\text { Intervalo } \\
\text { intercuartil }\end{array}$ & Mediana & Intervalo $_{\text {intercuartil }}$ & \\
\hline Energía (kcal/d) & 1127 & $816 ; 1344$ & 1448 & $1142 ; 1840$ & 0,000 \\
\hline Calcio (mg/d) & 625 & $374 ; 831$ & 813 & $486 ; 1136$ & 0,001 \\
\hline Hierro $(\mathrm{mg} / \mathrm{d})$ & 6,2 & 4,$8 ; 9,7$ & 6,2 & 4,$5 ; 8,8$ & NS \\
\hline Zinc (mg/d) & 5,2 & 3,$7 ; 7,9$ & 6,3 & 5,$0 ; 9,2$ & 0,018 \\
\hline Folatos ( $\mu$ gr/d) & 151 & $92 ; 197$ & 159 & $107 ; 227$ & NS \\
\hline Vitamina A ( $\mu$ gr RE/Dd) & 196 & $85 ; 533$ & 310 & $150 ; 582$ & 0,042 \\
\hline Proteínas & 44,1 & 34,$9 ; 59,1$ & 54,9 & 44,$6 ; 69,4$ & 0,000 \\
\hline
\end{tabular}

polenta, harina de maíz, estofados), milanesas, tartas, ensaladas, arroz, churrascos, fideos. Respecto a las bebidas se recurría a las gaseosas más económicas del mercado, al igual que a jugos para preparar. Sin embargo, al indagar acerca de la preparación de las comidas, en el área de producción de bienes y servicios, los entrevistados refirieron el consumo de ciertas comidas en general, sin proporcionar detalles sobre ingredientes, formas de preparación y frecuencia de consumo, mientras que en el área de producción primaria se observó un mayor énfasis en detallar qué elementos integraban cada plato. Asimismo, en este grupo se mencionó la incorporación en la comida diaria de un mayor volumen de verduras y carnes en las preparaciones y el consumo de frutas y dulces, no referidos en el área de producción de bienes y servicios.

Otra diferencia observable se dio con relación a la forma de preparación. En el área de producción de bienes y servicios, en general, las comidas se preparaban en ollas, hervidas o fritas, con escaso uso del horno. En cambio en el área de producción primaria se mencionó mayor consumo de alimentos crudos, uso de horno para cocinar y la cocción de carne a las brasas.

Las madres de ambos grupos coincidieron en señalar que los niños "pueden comer de todo", "lo que les gusta", puntualizando el consumo de frutas, yogures, leche y "siempre, algo con carne". En el área de producción primaria destacaron la importancia del consumo de verduras y cereales, ya que "tienen hierro" y "vitaminas" y la necesidad de realizar "las cuatro comidas". En cuanto a las restricciones del consumo de ciertos alimentos, las madres de este grupo explicaron la necesidad de disminuir o excluir alimentos muy salados y/o picantes, embutidos, alimentos fritos o muy grasos en niños pequeños. En cambio, en el área de producción de bienes y servicios se encontró que se restringía el consumo de alimentos dulces y "comida chatarra".

\section{DISCUSIÓN}

En el estudio, los niños que habitaban en zonas de producción primaria (agrícola- 
ganadera) mostraron un mejor estado nutricional que aquellos que vivían en áreas de producción de bienes y servicios.

La prevalencia de anemia y de deficiencia de hierro de los niños pertenecientes al área de producción primaria fueron comparables a los reportados a nivel provincial y nacional por la $\mathrm{ENNyS}^{(16)}$, mientras que los resultados de los niños del área de producción de bienes y servicios fueron superiores. También se halló asociación entre la deficiencia de hierro y el sexo del niño, observando que el sexo masculino presenta mayor prevalencia de deficiencia. La asociación entre sexo y anemia ha sido descripta en distintos países ${ }^{(24,25,26)}$ y podría indicar mayores requerimientos de hierro para los niños $(0,9 \mathrm{mg} / \mathrm{d})$ que para las niñas, en especial, durante la segunda mitad de la lactancia ${ }^{(27)}$.

En el presente estudio no se encontraron diferencias en el estado nutricional del zinc entre los grupos. El déficit en zinc fue similar al hallado por otro estudio, en niños de 1 a 2 años de familias receptoras del Plan Más Vida (PMV) de la provincia de Buenos Aires ${ }^{(28)}$.

Las diferencias halladas también podrían ser explicadas considerando las características propias de cada región. Es probable que el grupo del área de producción primaria resulte favorecido por situarse en un contexto geográfico cercano a las actividades de producción agrícola-ganadera, que haya facilitado a las familias el acceso y la disponibilidad de alimentos ricos en hierro. En este grupo destacaron la importancia del consumo de verduras, cereales, la cría y consumo de gallinas y pollos. Algunos autores refirieron que los hogares que tenían producción de alimentos de granja (verduras, frutas, huevo y aves de corral), tuvieron una mayor diversidad en la dieta y un mayor consumo de alimentos ricos en micronutrientes ${ }^{(7,29,30)}$.

Esta ventaja no se ve reflejada en mejores condiciones económicas sino -como surgió del análisis de las entrevistas- en la posibilidad de obtener los alimentos a través de estrategias como el trueque, canje (carnes en general) y regalos de familiares y vecinos, jugando un rol importante en el acceso y la disponibilidad de los alimentos. Esta interacción con individuos fuera de la familia, permitió ampliar la red social informal y los recursos disponibles. Estos resultados se encuentran en concordancia con otros estudios realizados en Brasil, que destacan la existencia de una red de cooperación vecinal y familiar para garantizar los alimentos mínimos en los hogares de bajos recursos económicos ${ }^{(31,32,33)}$.

Los resultados de la encuesta alimentaria muestran que la ingesta de energía y nutrientes fue superior en el área de producción primaria, aunque no se encontraron diferencias estadísticamente significativas con relación a la ingesta de hierro. Sin embargo, en ambos grupos la mediana del consumo de hierro fue inferior a lo recomendado por las agencias internacionales ${ }^{(34)}$. Nolan et al. ${ }^{(35)}$, en un estudio en niños de bajo nivel socioeconómico, con edades entre 3 y 24 meses, han reportado que el consumo de energía excedió las recomendaciones, al igual que en otros países en desarrollo, como Ghana, Guatemala y México ${ }^{(35,36)}$, no así el consumo de hierro, lo cual coincide con nuestros resultados. Una ingesta inadecuada de zinc de manera habitual es la causa más probable de deficiencia de $z_{\text {inc }}{ }^{(37)}$. Sin embargo, en este estudio, el consumo de zinc en ambos grupos poblacionales fue adecuado ${ }^{(34)}$. Es posible que la encuesta alimentaria sea una herramienta limitada en la evaluación del consumo, y haya estimado con poca precisión la ingesta ${ }^{(38)}$.

Otro factor que juega un rol importante en la calidad de la dieta es el nivel educativo materno. Darapheak et al. hallaron que los niños cuyas madres habían alcanzado los niveles más altos de educación tenían una mayor diversidad en la dieta, con un mayor consumo de carnes y vegetales, reflejado en un mejor estado nutricional, con relación a las madres con menos años de educación ${ }^{(7)}$. El análisis cualitativo muestra diferencias en cuanto a la preparación de los alimentos: las madres del área de producción primaria, con mayor nivel educativo, refirieron mayor detalle en la preparación de las comidas y destacaron la importancia del consumo de frutas y verduras para una alimentación adecuada. Estos dos factores quizás favorecieron una 
dieta de mayor calidad nutricional, que se vio reflejada en las menores prevalencias halladas. Asimismo, varios estudios coinciden en que un mayor nivel de instrucción materno tendría un efecto protector sobre el riesgo de desarrollo de anemia en sus hijos ${ }^{(39,40)}$.

La evaluación nutricional por antropometría mostró que los problemas nutricionales más frecuentes en ambos grupos fueron la baja talla y la obesidad, cuyo origen podría vincularse al acceso a ciertos tipos de alimentos más económicos pero cuantitativamente y cualitativamente inadecuados. Este fenómeno coincide con otros estudios que hallaron resultados similares ${ }^{(8,16,28,41)}$. Una vez más se expresa la transición epidemiológica nutricional en la coexistencia de baja talla con sobrepeso y obesidad, en diferentes áreas del interior de la provincia de Buenos Aires.

Una limitación del estudio fue no relevar la presencia de enteroparasitosis que pudieran afectar la ingesta alimentaria, la digestión, y la absorción de micronutrientes ${ }^{(42)}$. El estudio de Orden et al. ${ }^{(43)}$ mostró que la mayor concentración de parásitos hallados en áreas suburbanas de la provincia de Buenos Aires está caracterizada por las condiciones socioambientales más desfavorables, sugiriendo el impacto negativo de la urbanización, cuando no está acompañada de la provisión de servicios de infraestructura básicos. En el área de producción de bienes y servicios, la falta de agua corriente y desagües cloacales podría favorecer el desarrollo de estas infecciones pudiendo explicar, en parte, las altas prevalencias de anemia y deficiencia de hierro halladas.

En el presente estudio no se hallaron diferencias en la prevalencia de anemia y deficiencia de hierro entre los niños con y sin necesidades básicas insatisfechas. Quizás a través de otro abordaje de la situación económica de ambas poblaciones se hubiera podido encontrar alguna diferencia en indicadores de pobreza enmascarada entre los grupos ${ }^{(44)}$.

Aunque el muestreo fue intencional, permitió obtener datos básicos y tendencias de las variables de interés. La fortaleza del estudio fue sumar el enfoque cualitativo al análisis tradicional del estado nutricional, lo que permitió profundizar en los aspectos contextuales que influyen en la alimentación de los niños. Este tipo de abordaje integral resulta fundamental para el diseño de intervenciones nutricionales que permitan optimizar recursos y acciones en función de las necesidades como, por ejemplo, una educación nutricional que contribuya a fomentar buenos hábitos de alimentación y promover la comensalidad en el hogar, teniendo en cuenta las pautas culturales de la población y las actividades productivas del contexto.

\section{CONCLUSIONES}

Los niños del área de producción primaria relacionada con la agricultura y la ganadería presentaron una menor prevalencia de anemia y deficiencia de hierro que los niños del área de producción de bienes y servicios. Asimismo, tuvieron un consumo superior de energía, calcio, zinc, vitamina A, proteínas y una mayor diversidad y calidad en el consumo de alimentos.

El contexto geográfico de las familias que residen cerca de las fuentes de producción primaria, favorecería la interacción con individuos fuera de la familia, ampliando la red social informal y el acceso a los alimentos de mejor calidad nutricional. 


\section{AGRADECIMIENTOS}

Los autores agradecen a las autoridades municipales de cada localidad que autorizaron la realización del estudio, al equipo de salud de cada centro de salud que colaboró con la evaluación de los niños, a los padres o responsables de los niños y a los niños, por la participación en el estudio. Agradecemos la colaboración de los bioquímicos Andrea Tournier, Daniel Vogliolo y Silvia Pereyras, por el análisis de las muestras. A Bibiana Orden, por correcciones previas del manuscrito. Agradecemos también a los organismos que financiaron parte de la investigación: Ministerio de Salud y Ambiente de la Nación, Comisión Nacional Salud Investiga (año 2007) y Consejo Federal de Inversiones (años 2007-2009).

\section{REFERENCIAS BIBLIOGRÁFICAS}

1. Food and Agriculture Organization, World Health Organization. Documento final de la Conferencia: Declaración de Roma sobre la Nutrición [Internet]. 2014 [citado 3 dic 2015]. Disponible en: http://goo.gl/D5hlfD.

2. Aguirre P. Aspectos socioantropológicos de la obesidad en la pobreza. En: Peña M, Bacallao J (eds). La obesidad en la pobreza: un nuevo reto para la salud pública. Washington DC: OPS; 2000. p. 13-25.

3. Fotso JC. Child health inequities in developing countries: differences across urban and rural areas. International Journal of Equity in Health. 2006; 5:9. doi: 10.1186/1475-9276-5-9

4. Black RE, Victora CG, Walker SP, Bhutta ZA, Christian P, De Onis $M$, et al. Maternal and child undernutrition and overweight in low-income and middle-income countries. The Lancet. 2013;382(9890):427-451.

5. Kennedy G, Nantel G, Brouwer ID, Kok FJ. Does living in an urban environment confer advantages for childhood nutritional status?: Analysis of disparities in nutritional status by wealth and residence in Angola, Central African Republic and Senegal. Public Health Nutrition. 2006;9(2):187-193.

6. Yang W, Li X, Li Y, Zhang S, Liu L, Wang X, $\mathrm{Li}$ W. Anemia, malnutrition and their correlations with socio-demographic characteristics and feeding practices among infants aged 0-18 months in rural areas of Shaanxi province in northwestern China: a cross-sectional study. BMC Public Health. 2012;12:1127. doi: 10.1186/14712458-12-1127.
7. Darapheak C, Takano T, Kizuki M, Nakamura K, Seino K. Consumption of animal source foods and dietary diversity reduce stunting in children in Cambodia. International Archives of Medicine. 2013;6(1):29. doi: 10.1186/1755-7682-6-29.

8. Oyhenart EE, Torres MF, Quintero FA, Luis MA, Cesani MF, Zucchi M, Orden A. Estado nutricional y composición corporal de niños pobres residentes en barrios periféricos de La Plata, Argentina. Revista Panamericana de Salud Pública. 2007;22:194-201.

9. Olack B, Burke H, Cosmas L, Bamrah S, Dooling K, Feikin DR, Talley LE, Breiman RF. Nutritional status of under-five children living in an informal urban settlement in Nairobi, Kenya. Journal of Health, Population and Nutrition. 2011;29(4):357-363.

10. Srinivasan CS, Zanello G, Shankar B. Ruralurban disparities in child nutrition in Bangladesh and Nepal. BMC Public Health. 2013;14(13):581. doi: 10.1186/1471-2458-13-581.

11. Gutiérrez JL, López Ortega M. El impacto de los determinantes sociales de la salud en los niños. Boletín Médico del Hospital Infantil de México. 2014;71(2):117-125.

12. UNICEF. Child poverty in perspective: An overview of child well-being in rich countries, Innocenti Report Card 7 [Internet]. Florence: UNICEF Innocenti Research Centre; 2007 [citado 23 sep 2015]. Disponible en: http://goo.gl/kA9EY8.

13. Moonesinghe R, Bouye K, Penman-Aguilar A. Difference in health inequity between two population groups due to a social determinant of health. International Journal of Environmental Research and Public Health. 2014;11(12):13074-13083.

14. Ministerio de Economía, Dirección Provincial de Estudios y Proyecciones Económicas. Panorama productivo de la provincia de Buenos Aires [Internet]. 2012 [citado 23 sep 2015]. Disponible en: http://goo.gl/RdMfVp.

15. Quesada Aramburú J, Cadelli E. Hacia una clasificación de los municipios bonaerenses [Internet]. Ministerio de Economía, Dirección Provincial de Estudios y Proyecciones Económicas; 2012 [citado 23 sep 2015]. Disponible en: http://goo.gl/LImAQs.

16. Ministerio de Salud. Encuesta Nacional de Nutrición y Salud: Documento de Resultados 2007 [Internet]. 2007 [citado 1 dic 2015]. Disponible en: http://goo.gl/FkRk01.

17. World Health Organization. Iron deficiency anaemia: assessment, prevention and control: a 
guide for programme managers (WHO/NHD/01.3). Geneva: WHO; 2001.

18. International Zinc Nutrition Consultative Group (IZiNCG), Brown KH, Rivera JA, Bhutta Z, Gibson RS, King JC, Lönnerdal B, Ruel MT, Sandtröm B, Wasantwisut E, Hotz C. International Zinc Nutrition Consultative Group (IZiNCG) technical document \#1: Assessment of the risk of zinc deficiency in populations and options for its control. Food and Nutrition Bulletin. 2004;25(suppl 2):S99-S203.

19. World Health Organization. WHO child growth standards: Methods and development [Internet]. Geneva: WHO; 2006 [citado 11 dic 2015]. Disponible en: http://goo.gl/Y2K9BB.

20. Habicht JP. Estandarización de métodos epidemiológicos cuantitativos sobre el terreno. Boletín de la Oficina Sanitaria Panamericana. 1974;76(5):375-384.

21. Gibson RS. Principles of nutritional assessment. 2nd Ed. New York: Oxford University Press; 2005.

22. U.S. Department of Agriculture, Agricultural Research Service. Composition of Foods Raw, Processed, Prepared: USDA National Nutrient Database for Standard Reference, Release 17 [Internet]. 2004 [citado 10 sep 2015]. Disponible en: https://goo.gl/OSnXlq.

23. Instituto Nacional de Estadística y Censos. Acerca del método utilizado para la medición de la pobreza en Argentina [Internet]. 2003 [citado 10 sep 2015]. Disponible en: http://goo.gl/ArrUKq.

24. Domellof $M$, Lonnerdal B, Dewey KG, Cohen RJ, Rivera LL, Hernell O. Sex differences in iron status during infancy. Pediatrics. 2002; 110:545-552.

25. Morasso MC, Molero J, Vinocur P, Acosta L, Paccussi N, Raselli S, Falivene G, Viteri FE. Iron and vitamin A deficiencies and prevalence of anemia in boys and girls between 6 to 24 months of age in Chaco, Argentina. Archivos Latinoamericanos de Nutrición. 2003;53(1);21-27.

26. Lei QL, Dai BT, Xian Y, Yu J. Risk factors for nutritional iron deficiency anemia in children. Chinese Journal of Contemporary Pediatrics. 2014;16(1):16-19.

27. Wieringa FT, Berger J, Dijkhuizen MA, Hidayat A, Ninh NX, Utomo B, Wasantwisut E, Winichagoon P. Sex differences in prevalence of anaemia and iron deficiency in infancy in a large multi-country trial in South-East Asia. British Journal of Nutrition. 2007;98(5):1070-1076.
28. Varea A, Malpeli A, Etchegoyen G, Vojkovic M, Disalvo L, Apezteguía M, Pereyras S, Pattín J, Ortale S, Carmuega E, González HF. Short-term evaluation of the impact of a food program on the micronutrient nutritional status of Argentinean children under the age of six. Biological Trace Element Research. 2011;143(3):1337-1348.

29. Olney DK, Talukder A, lannotti LL, Ruel MT, Quinn V. Assessing impact and impact pathways of a homestead food production program on household and child nutrition in Cambodia. Food and Nutrition Bulletin. 2009;30(4):355-369.

30. Olney DK, Vicheka S, Kro M, Chakriya C, Kroeun H, Hoing LS, Talukder A, Quinn V, lannotti L, Becker E, Roopnaraine $T$. Using program impact pathways to understand and improve program delivery, utilization, and potential for impact of Helen Keller International's homestead food production program in Cambodia. Food and Nutrition Bulletin. 2013;34(2):169-184.

31. Tonial S. Desnutrição e obesidade: faces contraditórias na miséria e na abundância. Recife: Instituto Materno Infantil de Pernambuco; 2001.

32. Gerhardt TE. Situações de vida, pobreza e saúde: estratégias alimentares e práticas sociais no meio urbano. Ciência \& Saúde Coletiva. 2003;8(3):713-726.

33. Beraldo NA. Agricultores quilombolas, mediadores sociais e segurança alimentar: uma análise a partir das condições e estratégias de acesso aos alimentos da comunidade Maçambique/RS. [Dissertação de mestrado]. Porto Alegre: UFRGS; 2006.

34. Institute of Medicine. Dietary reference intakes: the essential guide to nutrient: The Essential Guide to Nutrient Requirements. Washington DC: National Academies Press; 2006.

35. Nolan K, Schell LM, Stark AD, Gómez MI. Longitudinal study of energy and nutrient intakes for infants from low-income, urban families. Public Health Nutrition. 2002;5(3):405-412.

36. Lutter CK, Rivera J. Nutritional status of infants and young children and characteristics of their diets. Journal of Nutrition. 2003;133(9):2941S2949S.

37. Grandy G, Weisstaub G, Lopez RD. Deficiencia de hierro y zinc en niños. Revista de la Sociedad Boliviana de Pediatría. 2010;49(1):25-31.

38. Arija Val V, Fernández Ballart J. Métodos de valoración del consumo alimentario. En: SalasSalvadó J, Bonada i Sanjaume A, Trallero Casañas R, Saló Solá E, Burgos Peláez R (ed). Nutrición 
y Dietética Clínica. 2a ed. Barcelona: Elsevier Masson; 2008. p. 65-82.

39. Agho KE, Dibley MJ, D’Este C, Gibberd R. Factors associated with haemoglobin concentration among Timor-Leste children aged 6-59 months. Journal of Health Population and Nutrition. 2008;26(2):200-209.

40. Ngesa O, Mwambi H. Prevalence and risk factors of anaemia among children aged between 6 months and 14 years in Kenya. PLoS One. 2014;9(11):e113756

41. Uauy R, Albala C, Kain J. Obesity trends in Latin America: transiting from under- to overweight. Journal of Nutrition. 2001;131(3):893S-899S.
42. Hesham MS, Edariah AB, Norhayati M. Intestinal parasitic infections and micronutrient deficiency: a review. Medical Journal of Malaysia. 2004;59(2):284-293.

43. Orden $A B$, Apezteguía $M C$, Ciarmela $M L$, Molina N, Pezzani B, Rosa D, Minvielle M. Nutritional status in parasitized and non-parasitized children from two districts of Buenos Aires, Argentina. American Journal of Human Biology. 2014;26(1):73-79.

44. Feres JC, Mancero X. El método de las necesidades básicas insatisfechas (NBI) y sus aplicaciones en América Latina. Santiago de Chile: Cepal; 2001.

\section{FORMA DE CITAR}

Pasarin L, Falivene MA, Disalvo L, Varea A, Apezteguía MC, Malpeli A, Sala M, González HF. Estudio cuali-cuantitativo del estado nutricional y alimentación en niños de 1 a 3 años de familias de bajos recursos en dos grupos poblacionales con diferentes actividades productivas (Buenos Aires, Argentina), 2007-2008. Salud Colectiva. 2016;12(2):239-250. doi: $10.18294 /$ sc. 2016.740 .

Recibido: 29 de septiembre de 2015 | Versión final: 17 de diciembre de 2015 | Aprobado: 15 de marzo de 2016

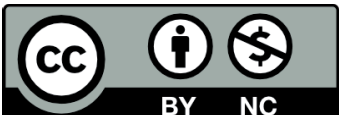
Internacional. Reconocimiento - Permite copiar, distribuir y comunicar públicamente la obra. A cambio, se debe reconocer y citar al autor original. No Comercial - Esta obra no puede ser utilizada con finalidades comerciales, a menos que se obtenga el permiso. 